\title{
Peripheral is Central to the question
}

\author{
Molecular Psychiatry (2005) 10, 421-422. \\ doi:10.1038/sj.mp.4001674
}

For the last several decades, our field has intensively searched for peripheral markers of psychiatric disorders, without success. Residual efforts in this area continue to be carried out by a few groups, as exemplified by the recent report on the correlation of peripheral-type benzodiazepine receptors with degrees of anxiety. ${ }^{1}$ However, in the vast majority of cases, we have not been able to ascertain with an acceptable degree of certainty the extent to which the periphery is involved in-or at least reflects - disturbances of central functions. The discovery of any significant peripheral-central connection in psychiatry has been elusive. Frustration and failure seem to have colluded to generate our current modi operandi. Therefore, most of us have been working under an unstated and unproven pseudo-dogma: The principle that peripheral findings do not or cannot reflect what occurs in the central nervous system (CNS). This belief has pervaded the field and has undermined our ability to confidently use the powerful tools of contemporary biology in order to dissect the biology of psychiatric disorders through investigation of peripheral markers, particularly those measured in peripheral blood. ${ }^{2}$

Uncertainty related to the diagnosis of psychiatric disorders and the lack of biological markers have further complicated the already daunting task of inferring CNS pathophysiology from the study of post-mortem brain tissues and animal models. Further complications result from the fact that in most instances several decades and the use of multiple drugs and treatments separate disease onset and post-mortem brain collection. This scenario is further compounded by the existence of a plethora of nonreplicated studies in psychiatric genetics. We have now entrusted the field of brain imaging to carry the burden of discovery of pathophysiological mechanisms of psychiatric disorders. ${ }^{3}$ Unfortunately, imaging cannot at the present time dissect fundamental molecular mechanisms. It appears that during our quest to understand mental disorders we have silently and unintentionally severed the brain from the body!

The past decade has brought a wealth of technological advances to biomedical research; gene profiling is a prototypical high-throughput tool that has already changed the way we look at cancer classification. ${ }^{4}$ Owing to a variety of complex issues, those advances have been only slowly applied to psychiatry. The biggest hurdles have been the paucity of high-quality post-mortem brain tissue ${ }^{5}$ and the lack of universally accepted animal models. ${ }^{6}$ These are real roadblocks and we currently do not have reasonable solutions for those critical problems. The euphoria over the possibility of quickly harnessing the data generated by the conclusion of the human genome project $^{7,8}$ has been in suspended animation over the past 4 years. A pivotal question has lingered unanswered: When will psychiatry start to benefit from the wealth of human genome information in the postgenomic era?

In this issue of Molecular Psychiatry, Segman et $a l^{9}$ started to answer this question for us (see pages 500-513). They examined gene expression profiles of peripheral blood mononuclear cells (PBMCs) collected from trauma survivors, and identified a gene expression 'signature' for post-traumatic stress disorder (PTSD). Patients were studied immediately following a traumatic event, 1 month, and 4 months later. Their results provide evidence that patterns of gene expression profile in PBMCs can identify survivors who either persistently manifested full criteria for acute and chronic PTSD or remained healthy at follow-up.

Upon reading their work it seems clear why PTSD would be the ideal condition to attempt such a conceptually bold study: The traumatic event is clearly identifiable; therefore, sequential time course samples can be obtained and studied in a systematic way. It is particularly amazing that exemplar research design can emerge from and be directly related to an unstable sociopolitical milieu.

This work by Segman et $a l^{9}$ carries the promise to bring a Renaissance to the field. They have revisited the question of finding psychiatric correlates in peripheral blood and their rigorous experimental design and data analysis have propelled research in PTSD to the 21st century. Their landmark work has achieved two fundamental goals. First, they show that peripheral markers can be used to determine symptom/disease outcome in psychiatry when they are meticulously analyzed during disease onset. Second, it becomes evident from their findings that exploratory research is crucial for psychiatric research. Even though we have already studied at length the classic hypotheses that were developed after the introduction of psychopharmacology 50 years ago, our understanding of the pathophysiology of psychiatric disorders remains incomplete and fragmented. Thus, hypotheses-generating, exploratory projects are extremely timely and will certainly foster a revival in the field by bringing to our attention new and unexpected insights. 
The challenges in the field of psychiatry continue to be fabulously multifaceted and complex, but perhaps now we can see some light at the end of this long tunnel. The work by Segman et al. ${ }^{9}$ has shown us where to look for: during disease onset and acute phase of symptom presentation. One can speculate that after the phase of active symptoms, during the period of predominant residual or sequela symptoms, peripheral transcriptional events may not be so evident or characteristic, which would make the identification of patterns of gene regulation and peripheral correlates a daunting task.

Suddenly, the future of psychiatric research appears brighter-we no longer have to procure postmortem brain tissue as our only source of human reference tissue. Let us hope that other investigators will soon follow the lead brought by this work and in this process we will help to bring mental research into the postgenomic era.
M-L Wong and J Licinio Center for Pharmacogenomics and Clinical Pharmacology, Neuropsychiatric Institute and David Geffen School of Medicine at University of California, Los Angeles, CA, USA E-mail: mali@ucla.edu

1 Nakamura K, Fukunishi I, Nakamoto Y, Iwahashi K, Yoshii M. Psychopharmacology (Berl) 2002; 162: 301-303.

2 Boulenger JP, Zarifian E. Rev Epidemiol Sante Publique 1985; 33: 361-367.

3 Frankle WG, Laruelle M. Ann Nucl Med 2002; 16: 437-446.

4 Lakhani SR, Ashworth A. Nat Rev Cancer 2001; 1: 151-157.

5 Mirnics K, Pevsner J. Nat Neurosci 2004; 7: 434-439.

6 Wong ML, Licinio J. Nat Rev Drug Discov 2004; 3: 136-151.

7 Venter JC, Adams MD, Myers EW, Li PW, Mural RJ, Sutton GG et al. Science 2001; 291: 1304-1351.

8 Lander ES, Linton LM, Birren B, Nusbaum C, Zody MC, Baldwin J et al. Nature 2001; 409: 860-921.

9 Segman RH, Shefi N, Goltser-Dubner T, Friedman N, Kaminski N, Shalev AY. Mol Psychiatry 2005; 10: 500-513. 